\title{
Implicit Function Theorem. Part II
}

\author{
Kazuhisa Nakasho \\ Yamaguchi University \\ Yamaguchi, Japan
}

\author{
Yasunari Shidama \\ Shinshu University \\ Nagano, Japan
}

Summary. In this article, we formalize differentiability of implicit function theorem in the Mizar system [3], 1. In the first half section, properties of Lipschitz continuous linear operators are discussed. Some norm properties of a direct sum decomposition of Lipschitz continuous linear operator are mentioned here.

In the last half section, differentiability of implicit function in implicit function theorem is formalized. The existence and uniqueness of implicit function in 6] is cited. We referred to [10, [1], and [2] in the formalization.

\section{MSC: 26B10 47A05 47J07 53A07 03B35}

Keywords: implicit function theorem; Lipschitz continuity; differentiability; implicit function

MML identifier: NDIFF_9, version: 8.1.09 5.57.1355

\section{Properties of Lipschitz Continuous Linear Operators}

From now on $S, T, W, Y$ denote real normed spaces, $f, f_{1}, f_{2}$ denote partial functions from $S$ to $T, Z$ denotes a subset of $S$, and $i, n$ denote natural numbers.

Now we state the propositions:

(1) Let us consider real normed spaces $E, F$, a partial function $f$ from $E$ to $F$, a subset $Z$ of $E$, and a point $z$ of $E$. Suppose $Z$ is open and $z \in Z$ and $Z \subseteq \operatorname{dom} f$ and $f$ is differentiable in $z$. Then

(i) $f\lceil Z$ is differentiable in $z$, and

(ii) $f^{\prime}(z)=\left(f\lceil Z)^{\prime}(z)\right.$. 
Proof: Consider $N$ being a neighbourhood of $z$ such that $N \subseteq \operatorname{dom} f$ and there exists a rest $R$ of $E, F$ such that for every point $x$ of $E$ such that $x \in N$ holds $f_{/ x}-f_{/ z}=\left(f^{\prime}(z)\right)(x-z)+R_{/ x-z}$. Consider $r$ being a real number such that $r>0$ and $\operatorname{Ball}(z, r) \subseteq Z$. Reconsider $N_{4}=N \cap Z$ as a neighbourhood of $z$. Consider $R$ being a rest of $E, F$ such that for every point $x$ of $E$ such that $x \in N$ holds $f_{/ x}-f_{/ z}=\left(f^{\prime}(z)\right)(x-z)+R_{/ x-z}$. For every point $x$ of $E$ such that $x \in N_{4}$ holds $\left(f\lceil Z)_{/ x}-\left(f\lceil Z)_{/ z}=\right.\right.$ $\left(f^{\prime}(z)\right)(x-z)+R_{/ x-z}$.

(2) Let us consider real normed spaces $E, F, G$, a partial function $f$ from $E \times F$ to $G$, a subset $Z$ of $E \times F$, and a point $z$ of $E \times F$. Suppose $Z$ is open and $z \in Z$ and $Z \subseteq \operatorname{dom} f$. Then

(i) if $f$ is partially differentiable in $z$ w.r.t. 1 , then $f\lceil Z$ is partially differentiable in $z$ w.r.t. 1 and partdiff $(f, z)$ w.r.t. $1=$ $\operatorname{partdiff}(f\lceil Z, z)$ w.r.t. 1 , and

(ii) if $f$ is partially differentiable in $z$ w.r.t. 2 , then $f\lceil Z$ is partially differentiable in $z$ w.r.t. 2 and partdiff $(f, z)$ w.r.t. $2=$ $\operatorname{partdiff}(f\lceil Z, z)$ w.r.t. 2 .

Proof: If $f$ is partially differentiable in $z$ w.r.t. 1 , then $f\lceil Z$ is partially differentiable in $z$ w.r.t. 1 and partdiff $(f, z)$ w.r.t. $1=\operatorname{partdiff}(f\lceil Z, z)$ w.r.t. 1 . Set $g=f \cdot(\operatorname{reproj} 2(z))$. Consider $N$ being a neighbourhood of $(z)_{2}$ such that $N \subseteq \operatorname{dom} g$ and there exists a rest $R$ of $F, G$ such that for every point $x$ of $F$ such that $x \in N$ holds $g_{/ x}-g_{/(z)_{2}}=($ partdiff $(f, z)$ w.r.t. 2$)(x-$ $\left.(z)_{2}\right)+R_{/ x-(z)_{2}}$. Consider $R$ being a rest of $F, G$ such that for every point $x$ of $F$ such that $x \in N$ holds $g_{/ x}-g_{/(z)_{2}}=($ partdiff $(f, z)$ w.r.t. 2$)(x-$ $\left.(z)_{2}\right)+R_{/ x-(z)_{2}}$.

Set $h=\left(f\lceil Z) \cdot(\operatorname{reproj} 2(z))\right.$. Consider $r_{1}$ being a real number such that $r_{1}>0$ and $\operatorname{Ball}\left(z, r_{1}\right) \subseteq Z$. Consider $r_{2}$ being a real number such that $r_{2}>0$ and $\left\{y\right.$, where $y$ is a point of $\left.F:\left\|y-(z)_{2}\right\|<r_{2}\right\} \subseteq N$. Set $r=\min \left(r_{1}, r_{2}\right)$. Set $M=\operatorname{Ball}\left((z)_{2}, r\right) . M \subseteq N$ and for every point $x$ of $F$ such that $x \in M$ holds $(\operatorname{reproj} 2(z))(x) \in Z$. $M \subseteq$ dom $h$. For every point $x$ of $F$ such that $x \in M$ holds $h_{/ x}-h_{/(z)_{2}}=(\operatorname{partdiff}(f, z)$ w.r.t. 2$)(x-$ $\left.(z)_{2}\right)+R / x-(z)_{2}$.

(3) Let us consider real normed spaces $X, E, G, F$, a bilinear operator $B$ from $E \times F$ into $G$, a partial function $f$ from $X$ to $E$, a partial function $g$ from $X$ to $F$, and a subset $S$ of $X$. Suppose $B$ is continuous on the carrier of $E \times F$ and $S \subseteq \operatorname{dom} f$ and $S \subseteq \operatorname{dom} g$ and for every point $s$ of $X$ such that $s \in S$ holds $f$ is continuous in $s$ and for every point $s$ of $X$ such that $s \in S$ holds $g$ is continuous in $s$. Then there exists a partial function $H$ from $X$ to $G$ such that 
(i) $\operatorname{dom} H=S$, and

(ii) for every point $s$ of $X$ such that $s \in S$ holds $H(s)=B(f(s), g(s))$, and

(iii) $H$ is continuous on $S$.

Proof: Define $\mathcal{P}$ [object, object] $\equiv$ there exists a point $t$ of $X$ such that $t=\$_{1}$ and $\$_{2}=B(f(t), g(t))$. For every object $x$ such that $x \in S$ there exists an object $y$ such that $y \in$ the carrier of $G$ and $\mathcal{P}[x, y]$. Consider $H$ being a function from $S$ into $G$ such that for every object $z$ such that $z \in S$ holds $\mathcal{P}[z, H(z)]$. For every point $s$ of $X$ such that $s \in S$ holds $H(s)=B(f(s), g(s))$. For every point $x_{0}$ of $X$ and for every real number $r$ such that $x_{0} \in S$ and $0<r$ there exists a real number $p_{2}$ such that $0<p_{2}$ and for every point $x_{1}$ of $X$ such that $x_{1} \in S$ and $\left\|x_{1}-x_{0}\right\|<p_{2}$ holds $\left\|H_{/ x_{1}}-H_{/ x_{0}}\right\|<r$.

(4) Let us consider real normed spaces $E, F$, a partial function $g$ from $E$ to $F$, and a subset $A$ of $E$. Suppose $g$ is continuous on $A$ and $\operatorname{dom} g=A$. Then there exists a partial function $x_{2}$ from $E$ to $E \times F$ such that

(i) $\operatorname{dom} x_{2}=A$, and

(ii) for every point $x$ of $E$ such that $x \in A$ holds $x_{2}(x)=\langle x, g(x)\rangle$, and (iii) $x_{2}$ is continuous on $A$.

Proof: Define $\mathcal{P}$ [object, object $] \equiv$ there exists a point $t$ of $E$ such that $t=\$_{1}$ and $\$_{2}=\langle t, g(t)\rangle$. For every object $x$ such that $x \in S$ there exists an object $y$ such that $y \in$ the carrier of $E \times F$ and $\mathcal{P}[x, y]$. Consider $H$ being a function from $S$ into $E \times F$ such that for every object $z$ such that $z \in S$ holds $\mathcal{P}[z, H(z)]$. For every point $s$ of $E$ such that $s \in S$ holds $H(s)=\langle s, g(s)\rangle$. For every point $x_{0}$ of $E$ and for every real number $r$ such that $x_{0} \in S$ and $0<r$ there exists a real number $p_{2}$ such that $0<p_{2}$ and for every point $x_{1}$ of $E$ such that $x_{1} \in S$ and $\left\|x_{1}-x_{0}\right\|<p_{2}$ holds $\left\|H_{/ x_{1}}-H_{/ x_{0}}\right\|<r$.

(5) Let us consider real normed spaces $S, T, V$, a point $x_{0}$ of $V$, a partial function $f_{1}$ from the carrier of $V$ to the carrier of $S$, and a partial function $f_{2}$ from the carrier of $S$ to the carrier of $T$. Suppose $x_{0} \in \operatorname{dom}\left(f_{2} \cdot f_{1}\right)$ and $f_{1}$ is continuous in $x_{0}$ and $f_{2}$ is continuous in $f_{1 / x_{0}}$. Then $f_{2} \cdot f_{1}$ is continuous in $x_{0}$.

Proof: $\operatorname{rng}\left(f_{1 *} s_{1}\right) \subseteq \operatorname{dom} f_{2}$.

(6) Let us consider real normed spaces $E, F$, a point $z$ of $E \times F$, a point $x$ of $E$, and a point $y$ of $F$. Suppose $z=\langle x, y\rangle$. Then $\|z\| \leqslant\|x\|+\|y\|$.

(7) Let us consider real normed spaces $E, F, G$, and a linear operator $L$ from $E \times F$ into $G$. Then there exists a linear operator $L_{1}$ from $E$ into $G$ 
and there exists a linear operator $L_{2}$ from $F$ into $G$ such that for every point $x$ of $E$ and for every point $y$ of $F, L(\langle x, y\rangle)=L_{1}(x)+L_{2}(y)$ and for every point $x$ of $E, L_{1}(x)=L_{/\left\langle x, 0_{F}\right\rangle}$ and for every point $y$ of $F$, $L_{2}(y)=L /\left\langle 0_{E}, y\right\rangle \cdot$

Proof: Define $\mathcal{C}$ (point of $E$ ) $=L_{/}\left\langle \$_{1}, 0_{F}\right\rangle$. Consider $L_{1}$ being a function from the carrier of $E$ into the carrier of $G$ such that for every point $x$ of $E, L_{1}(x)=\mathcal{C}(x)$. For every elements $s, t$ of $E, L_{1}(s+t)=L_{1}(s)+L_{1}(t)$. For every element $s$ of $E$ and for every real number $r, L_{1}(r \cdot s)=r \cdot L_{1}(s)$. Define $\mathcal{D}$ (point of $F$ ) $=L /\left\langle 0_{E}, \$_{1}\right\rangle$. Consider $L_{2}$ being a function from the carrier of $F$ into the carrier of $G$ such that for every point $x$ of $F$, $L_{2}(x)=\mathcal{D}(x)$. For every elements $s, t$ of $F, L_{2}(s+t)=L_{2}(s)+L_{2}(t)$. For every element $s$ of $F$ and for every real number $r, L_{2}(r \cdot s)=r \cdot L_{2}(s)$. For every point $x$ of $E$ and for every point $y$ of $F, L(\langle x, y\rangle)=L_{1}(x)+L_{2}(y)$.

(8) Let us consider real normed spaces $E, F, G$, a linear operator $L$ from $E \times F$ into $G$, a linear operator $L_{11}$ from $E$ into $G$, a linear operator $L_{12}$ from $F$ into $G$, a linear operator $L_{21}$ from $E$ into $G$, and a linear operator $L_{22}$ from $F$ into $G$. Suppose for every point $x$ of $E$ and for every point $y$ of $F, L(\langle x, y\rangle)=L_{11}(x)+L_{12}(y)$ and for every point $x$ of $E$ and for every point $y$ of $F, L(\langle x, y\rangle)=L_{21}(x)+L_{22}(y)$. Then

(i) $L_{11}=L_{21}$, and

(ii) $L_{12}=L_{22}$.

(9) Let us consider real normed spaces $E, F, G$, a linear operator $L_{1}$ from $E$ into $G$, and a linear operator $L_{2}$ from $F$ into $G$. Then there exists a linear operator $L$ from $E \times F$ into $G$ such that

(i) for every point $x$ of $E$ and for every point $y$ of $F, L(\langle x, y\rangle)=L_{1}(x)+$ $L_{2}(y)$, and

(ii) for every point $x$ of $E, L_{1}(x)=L_{/\left\langle x, 0_{F}\right\rangle}$, and

(iii) for every point $y$ of $F, L_{2}(y)=L /\left\langle 0_{E}, y\right\rangle$.

Proof: Define $\mathcal{P}$ [object, object] $\equiv$ there exists a point $x$ of $E$ and there exists a point $y$ of $F$ such that $\$_{1}=\langle x, y\rangle$ and $\$_{2}=L_{1}(x)+L_{2}(y)$. For every element $z$ of $E \times F$, there exists an element $y$ of $G$ such that $\mathcal{P}[z, y]$. Consider $L$ being a function from $E \times F$ into $G$ such that for every element $z$ of $E \times F, \mathcal{P}[z, L(z)]$. For every points $z, w$ of $E \times F$, $L(z+w)=L(z)+L(w)$. For every element $z$ of $E \times F$ and for every real number $r, L(r \cdot z)=r \cdot L(z)$. For every point $x$ of $E$ and for every point $y$ 
of $F, L(\langle x, y\rangle)=L_{1}(x)+L_{2}(y)$. For every point $x$ of $E, L_{1}(x)=L_{/\left\langle x, 0_{F}\right\rangle}$. For every point $y$ of $F, L_{2}(y)=L_{/\left\langle 0_{E}, y\right\rangle}$ by [9, (3)].

(10) Let us consider real normed spaces $E, F, G$, and a Lipschitzian linear operator $L$ from $E \times F$ into $G$. Then there exists a Lipschitzian linear operator $L_{1}$ from $E$ into $G$ and there exists a Lipschitzian linear operator $L_{2}$ from $F$ into $G$ such that for every point $x$ of $E$ and for every point $y$ of $F, L(\langle x, y\rangle)=L_{1}(x)+L_{2}(y)$ and for every point $x$ of $E, L_{1}(x)=L_{/\left\langle x, 0_{F}\right\rangle}$ and for every point $y$ of $F, L_{2}(y)=L_{/\left\langle 0_{E}, y\right\rangle}$. The theorem is a consequence of $(7)$.

(11) Let us consider real normed spaces $E, F, G$, a Lipschitzian linear operator $L_{1}$ from $E$ into $G$, and a Lipschitzian linear operator $L_{2}$ from $F$ into $G$. Then there exists a Lipschitzian linear operator $L$ from $E \times F$ into $G$ such that

(i) for every point $x$ of $E$ and for every point $y$ of $F, L(\langle x, y\rangle)=L_{1}(x)+$ $L_{2}(y)$, and

(ii) for every point $x$ of $E, L_{1}(x)=L_{/\left\langle x, 0_{F}\right\rangle}$, and

(iii) for every point $y$ of $F, L_{2}(y)=L_{/\left\langle 0_{E}, y\right\rangle}$.

The theorem is a consequence of (9).

(12) Let us consider real normed spaces $E, F, G$, and a point $L$ of the real norm space of bounded linear operators from $E \times F$ into $G$. Then there exists a point $L_{1}$ of the real norm space of bounded linear operators from $E$ into $G$ and there exists a point $L_{2}$ of the real norm space of bounded linear operators from $F$ into $G$ such that for every point $x$ of $E$ and for every point $y$ of $F, L(\langle x, y\rangle)=L_{1}(x)+L_{2}(y)$ and for every point $x$ of $E$, $L_{1}(x)=L\left(\left\langle x, 0_{F}\right\rangle\right)$ and for every point $y$ of $F, L_{2}(y)=L\left(\left\langle 0_{E}, y\right\rangle\right)$ and $\|L\| \leqslant\left\|L_{1}\right\|+\left\|L_{2}\right\|$ and $\left\|L_{1}\right\| \leqslant\|L\|$ and $\left\|L_{2}\right\| \leqslant\|L\|$.

Proof: Reconsider $L=L_{4}$ as a Lipschitzian linear operator from $E \times$ $F$ into $G$. Consider $L_{1}$ being a Lipschitzian linear operator from $E$ into $G, L_{2}$ being a Lipschitzian linear operator from $F$ into $G$ such that for every point $x$ of $E$ and for every point $y$ of $F, L(\langle x, y\rangle)=L_{1}(x)+L_{2}(y)$ and for every point $x$ of $E, L_{1}(x)=L_{/\left\langle x, 0_{F}\right\rangle}$ and for every point $y$ of $F$, $L_{2}(y)=L_{/\left\langle 0_{E}, y\right\rangle} \cdot$

Reconsider $L_{5}=L_{1}$ as a point of the real norm space of bounded linear operators from $E$ into $G$. Reconsider $L_{3}=L_{2}$ as a point of the real norm space of bounded linear operators from $F$ into $G$. For every point $x$ of $E, L_{5}(x)=L_{4}\left(\left\langle x, 0_{F}\right\rangle\right)$. For every point $y$ of $F, L_{3}(y)=L_{4}\left(\left\langle 0_{E}, y\right\rangle\right)$. For every real number $t$ such that $t \in \operatorname{PreNorms}(L)$ holds $t \leqslant\left\|L_{5}\right\|+\left\|L_{3}\right\|$. 
For every real number $t$ such that $t \in \operatorname{PreNorms}\left(L_{1}\right)$ holds $t \leqslant\left\|L_{4}\right\|$. For every real number $t$ such that $t \in \operatorname{PreNorms}\left(L_{2}\right)$ holds $t \leqslant\left\|L_{4}\right\|$.

(13) Let us consider real normed spaces $E, F, G$, a point $L$ of the real norm space of bounded linear operators from $E \times F$ into $G$, points $L_{11}, L_{12}$ of the real norm space of bounded linear operators from $E$ into $G$, and points $L_{21}, L_{22}$ of the real norm space of bounded linear operators from $F$ into $G$. Suppose for every point $x$ of $E$ and for every point $y$ of $F, L(\langle x$, $y\rangle)=L_{11}(x)+L_{21}(y)$ and for every point $x$ of $E$ and for every point $y$ of $F, L(\langle x, y\rangle)=L_{12}(x)+L_{22}(y)$. Then

(i) $L_{11}=L_{12}$, and

(ii) $L_{21}=L_{22}$.

The theorem is a consequence of (8).

\section{Differentiability of Implicit Function}

Now we state the propositions:

(14) Let us consider real normed spaces $E, G, F$, a subset $Z$ of $E \times F$, a partial function $f$ from $E \times F$ to $G$, and a point $z$ of $E \times F$. Suppose $f$ is differentiable in $z$. Then

(i) $f$ is partially differentiable in $z$ w.r.t. 1 , and

(ii) $f$ is partially differentiable in $z$ w.r.t. 2 , and

(iii) for every point $d_{7}$ of $E$ and for every point $d_{8}$ of $F,\left(f^{\prime}(z)\right)\left(\left\langle d_{7}\right.\right.$, $\left.\left.d_{8}\right\rangle\right)=($ partdiff $(f, z)$ w.r.t. 1$)\left(d_{7}\right)+(\operatorname{partdiff}(f, z)$ w.r.t. 2$)\left(d_{8}\right)$.

Proof: Reconsider $y=(\operatorname{IsoCPNrSP}(E, F))(z)$ as a point of $\Pi\langle E, F\rangle$. Consider $N$ being a neighbourhood of $z$ such that $N \subseteq \operatorname{dom} f$ and there exists a rest $R$ of $E \times F, G$ such that for every point $w$ of $E \times F$ such that $w \in N$ holds $f_{/ w}-f_{/ z}=\left(f^{\prime}(z)\right)(w-z)+R / w-z$. Consider $R$ being a rest of $E \times F, G$ such that for every point $w$ of $E \times F$ such that $w \in N$ holds $f_{/ w}-f_{/ z}=\left(f^{\prime}(z)\right)(w-z)+R_{/ w-z}$. Reconsider $L=f^{\prime}(z)$ as a Lipschitzian linear operator from $E \times F$ into $G$. Consider $L_{1}$ being a Lipschitzian linear operator from $E$ into $G, L_{2}$ being a Lipschitzian linear operator from $F$ into $G$ such that for every point $d_{7}$ of $E$ and for every point $d_{8}$ of $F, L\left(\left\langle d_{7}\right.\right.$, $\left.\left.d_{8}\right\rangle\right)=L_{1}\left(d_{7}\right)+L_{2}\left(d_{8}\right)$ and for every point $d_{7}$ of $E, L_{1}\left(d_{7}\right)=L_{/\left\langle d_{7}, 0_{F}\right\rangle}$ and for every point $d_{8}$ of $F, L_{2}\left(d_{8}\right)=L_{/\left\langle 0_{E}, d_{8}\right\rangle}$.

Reconsider $L_{3}=L_{1}$ as a point of the real norm space of bounded linear operators from $E$ into $G$. Reconsider $L_{4}=L_{2}$ as a point of the real norm space of bounded linear operators from $F$ into $G$. Set $g_{1}=f \cdot(\operatorname{reproj} 1(z))$. 
Set $g_{2}=f \cdot(\operatorname{reproj} 2(z))$. Reconsider $x=(z)_{1}$ as a point of $E$. Reconsider $y=(z)_{2}$ as a point of $F$. Consider $r_{0}$ being a real number such that $0<r_{0}$ and $\left\{y\right.$, where $y$ is a point of $\left.E \times F:\|y-z\|<r_{0}\right\} \subseteq N$. Consider $r$ being a real number such that $0<r<r_{0}$ and $\operatorname{Ball}(x, r) \times \operatorname{Ball}(y, r) \subseteq$ $\operatorname{Ball}\left(z, r_{0}\right)$. Define $\mathcal{C}$ (point of $\left.E\right)=R{ }_{/\left\langle \$_{1}, 0_{F}\right\rangle}$. Consider $R_{1}$ being a function from the carrier of $E$ into the carrier of $G$ such that for every point $p$ of $E, R_{1}(p)=\mathcal{C}(p)$. Define $\mathcal{D}$ (point of $\left.F\right)=R_{/\left\langle 0_{E}, \$_{1}\right\rangle}$. Consider $R_{2}$ being a function from the carrier of $F$ into the carrier of $G$ such that for every point $p$ of $F, R_{2}(p)=\mathcal{D}(p)$.

For every real number $r$ such that $r>0$ there exists a real number $d$ such that $d>0$ and for every point $z$ of $E$ such that $z \neq 0_{E}$ and $\|z\|<d$ holds $\|z\|^{-1} \cdot\left\|R_{1 / z}\right\|<r$. For every real number $r$ such that $r>0$ there exists a real number $d$ such that $d>0$ and for every point $z$ of $F$ such that $z \neq 0_{F}$ and $\|z\|<d$ holds $\|z\|^{-1} \cdot\left\|R_{2 / z}\right\|<r$. Reconsider $N_{1}=\operatorname{Ball}(x, r)$ as a neighbourhood of $x$. Reconsider $N_{2}=\operatorname{Ball}(y, r)$ as a neighbourhood of y. $N_{1} \subseteq \operatorname{dom} g_{1} . N_{2} \subseteq \operatorname{dom} g_{2}$. For every point $x_{1}$ of $E$ such that $x_{1} \in N_{1}$ holds $g_{1 / x_{1}}-g_{1 / x}=L_{3}\left(x_{1}-x\right)+R_{1 / x_{1}-x}$. For every point $y_{1}$ of $F$ such that $y_{1} \in N_{2}$ holds $g_{2 / y_{1}}-g_{2 / y}=L_{4}\left(y_{1}-y\right)+R_{2 / y_{1}-y}$.

(15) Let us consider real normed spaces $E, G, F$, a subset $Z$ of $E \times F$, a partial function $f$ from $E \times F$ to $G$, a point $a$ of $E$, a point $b$ of $F$, a point $c$ of $G$, a point $z$ of $E \times F$, real numbers $r_{1}, r_{2}$, a partial function $g$ from $E$ to $F$, a Lipschitzian linear operator $P$ from $E$ into $G$, and a Lipschitzian linear operator $Q$ from $G$ into $F$.

Suppose $Z$ is open and $\operatorname{dom} f=Z$ and $z=\langle a, b\rangle$ and $z \in Z$ and $f(a, b)=c$ and $f$ is differentiable in $z$ and $0<r_{1}$ and $0<r_{2}$ and $\operatorname{dom} g=$ $\operatorname{Ball}\left(a, r_{1}\right)$ and $\operatorname{rng} g \subseteq \operatorname{Ball}\left(b, r_{2}\right)$ and $g(a)=b$ and $g$ is continuous in $a$ and for every point $x$ of $E$ such that $x \in \operatorname{Ball}\left(a, r_{1}\right)$ holds $f(x, g(x))=c$ and partdiff $(f, z)$ w.r.t. 2 is one-to-one and $Q=(\operatorname{partdiff}(f, z) \text { w.r.t. } 2)^{-1}$ and $P=\operatorname{partdiff}(f, z)$ w.r.t. 1 . Then

(i) $g$ is differentiable in $a$, and

(ii) $g^{\prime}(a)=-Q \cdot P$.

Proof: Reconsider $L=Q \cdot P$ as a point of the real norm space of bounded linear operators from $E$ into $F$. Consider $N_{0}$ being a neighbourhood of $z$ such that $N_{0} \subseteq \operatorname{dom} f$ and there exists a rest $R$ of $E \times F, G$ such that for every point $w$ of $E \times F$ such that $w \in N_{0}$ holds $f_{/ w}-f_{/ z}=\left(f^{\prime}(z)\right)(w-$ $z)+R_{/ w-z}$. Consider $R$ being a rest of $E \times F, G$ such that for every point $w$ of $E \times F$ such that $w \in N_{0}$ holds $f_{/ w}-f_{/ z}=\left(f^{\prime}(z)\right)(w-z)+R_{/ w-z}$. Consider $r_{0}$ being a real number such that $0<r_{0}$ and $\{y$, where $y$ is a point of $\left.E \times F:\|y-z\|<r_{0}\right\} \subseteq N_{0}$. Consider $r_{3}$ being a real number 
such that $0<r_{3}<r_{0}$ and $\operatorname{Ball}\left(a, r_{3}\right) \times \operatorname{Ball}\left(b, r_{3}\right) \subseteq \operatorname{Ball}\left(z, r_{0}\right)$. Reconsider $r_{4}=\min \left(r_{1}, r_{3}\right)$ as a real number.

Consider $r_{5}$ being a real number such that $0<r_{5}$ and for every point $x_{1}$ of $E$ such that $x_{1} \in \operatorname{dom} g$ and $\left\|x_{1}-a\right\|<r_{5}$ holds $\left\|g_{/ x_{1}}-g_{/ a}\right\|<r_{3}$. Reconsider $r_{6}=\min \left(r_{4}, r_{5}\right)$ as a real number. Reconsider $N=\operatorname{Ball}\left(a, r_{6}\right)$ as a neighbourhood of $a$. Define $\mathcal{C}$ (point of $E)=-Q\left(R_{/\left\langle \$_{1}, g_{/ a+\Phi_{1}}-g_{/ a}\right\rangle}\right)$. Consider $R_{1}$ being a function from the carrier of $E$ into the carrier of $F$ such that for every point $p$ of $E, R_{1}(p)=\mathcal{C}(p)$. For every point $x$ of $E$ such that $x \in N$ holds $g_{/ x}-g_{/ a}=(-L)(x-a)+R_{1 / x-a}$. Define $\mathcal{D}$ [point of $E$, object $] \equiv \$_{2}=\left\langle \$_{1}, g_{/ a+\$_{1}}-g_{/ a}\right\rangle$. For every element $d_{7}$ of the carrier of $E$, there exists an element $d_{8}$ of the carrier of $E \times F$ such that $\mathcal{D}\left[d_{7}, d_{8}\right]$.

Consider $V$ being a function from the carrier of $E$ into the carrier of $E \times F$ such that for every element $d_{7}$ of the carrier of $E, \mathcal{D}\left[d_{7}, V\left(d_{7}\right)\right]$. Reconsider $Q_{1}=Q$ as a point of the real norm space of bounded linear operators from $G$ into $F$. Set $Q_{2}=\left\|Q_{1}\right\|$. Consider $d_{0}$ being a real number such that $d_{0}>0$ and for every point $d_{9}$ of $E \times F$ such that $d_{9} \neq 0_{E \times F}$ and $\left\|d_{9}\right\|<d_{0}$ holds $\left\|d_{9}\right\|^{-1} \cdot\left\|R / d_{9}\right\|<\frac{1}{2 \cdot\left(Q_{2}+1\right)}$. Consider $d_{1}$ being a real number such that $0<d_{1}<d_{0}$ and $\operatorname{Ball}\left(a, d_{1}\right) \times \operatorname{Ball}\left(g_{/ a}, d_{1}\right) \subseteq \operatorname{Ball}\left(z, d_{0}\right)$. Consider $d_{2}$ being a real number such that $0<d_{2}$ and for every point $x_{1}$ of $E$ such that $x_{1} \in \operatorname{dom} g$ and $\left\|x_{1}-a\right\|<d_{2}$ holds $\left\|g_{/ x_{1}}-g_{/ a}\right\|<d_{1}$. Reconsider $d_{3}=\min \left(d_{1}, d_{2}\right)$ as a real number. Reconsider $d_{4}=\min \left(d_{3}, r_{1}\right)$ as a real number.

For every point $d_{7}$ of $E$ such that $d_{7} \neq 0_{E}$ and $\left\|d_{7}\right\|<d_{4}$ holds $\left\|R_{/ V\left(d_{7}\right)}\right\| \leqslant \frac{1}{2 \cdot\left(Q_{2}+1\right)} \cdot\left(\left\|d_{7}\right\|+\left\|g_{/ a+d_{7}}-g_{/ a}\right\|\right)$. For every point $d_{7}$ of $E$ such that $d_{7} \neq 0_{E}$ and $\left\|d_{7}\right\|<d_{4}$ holds $\left\|R_{1 / d_{7}}\right\| \leqslant \frac{1}{2} \cdot\left(\left\|d_{7}\right\|+\left\|g_{/ a+d_{7}}-g_{/ a}\right\|\right)$. Set $Q_{3}=\|L\|$. Reconsider $d_{5}=\min \left(r_{6}, d_{4}\right)$ as a real number. For every point $d_{7}$ of $E$ such that $d_{7} \neq 0_{E}$ and $\left\|d_{7}\right\|<d_{5}$ holds $\left\|g_{/ a+d_{7}}-g_{/ a}\right\| \leqslant$ $\left(2 \cdot Q_{3}+1\right) \cdot\left\|d_{7}\right\|$. For every real number $r$ such that $r>0$ there exists a real number $d$ such that $d>0$ and for every point $d_{7}$ of $E$ such that $d_{7} \neq 0_{E}$ and $\left\|d_{7}\right\|<d$ holds $\left\|d_{7}\right\|^{-1} \cdot\left\|R_{1 / d_{7}}\right\|<r$ by [4, (23)], [7, (7)], [8, (18)].

From now on $X, Y, Z$ denote non trivial real Banach spaces.

Now we state the propositions:

(16) Let us consider a point $u$ of the real norm space of bounded linear operators from $X$ into $Y$. Suppose $u$ is invertible. Then there exist real numbers $K, s$ such that

(i) $0 \leqslant K$, and

(ii) $0<s$, and

(iii) for every point $d_{6}$ of the real norm space of bounded linear operators 
from $X$ into $Y$ such that $\left\|d_{6}\right\|<s$ holds $u+d_{6}$ is invertible and $\left\|\operatorname{Inv} u+d_{6}-\operatorname{Inv} u--(\operatorname{Inv} u) \cdot d_{6} \cdot(\operatorname{Inv} u)\right\| \leqslant K \cdot\left(\left\|d_{6}\right\| \cdot\left\|d_{6}\right\|\right)$.

(17) Let us consider a partial function $I$ from the real norm space of bounded linear operators from $X$ into $Y$ to the real norm space of bounded linear operators from $Y$ into $X$. Suppose $\operatorname{dom} I=\operatorname{InvertOpers}(X, Y)$ and for every point $u$ of the real norm space of bounded linear operators from $X$ into $Y$ such that $u \in \operatorname{InvertOpers}(X, Y)$ holds $I(u)=\operatorname{Inv} u$. Let us consider a point $u$ of the real norm space of bounded linear operators from $X$ into $Y$. Suppose $u \in \operatorname{InvertOpers}(X, Y)$. Then

(i) $I$ is differentiable in $u$, and

(ii) for every point $d_{6}$ of the real norm space of bounded linear operators from $X$ into $Y,\left(I^{\prime}(u)\right)\left(d_{6}\right)=-(\operatorname{Inv} u) \cdot d_{6} \cdot(\operatorname{Inv} u)$.

Proof: Set $S=$ the real norm space of bounded linear operators from $X$ into $Y$. Set $W=$ the real norm space of bounded linear operators from $Y$ into $X$. Set $N=\operatorname{InvertOpers~}(X, Y)$. Define $\mathcal{C}($ point of $S)=$ $-(\operatorname{Inv} u) \cdot \$_{1} \cdot(\operatorname{Inv} u)$. Consider $L$ being a function from the carrier of $S$ into the carrier of $W$ such that for every point $x$ of $S, L(x)=\mathcal{C}(x)$. For every elements $s, t$ of $S, L(s+t)=L(s)+L(t)$. For every element $s$ of $S$ and for every real number $r, L(r \cdot s)=r \cdot L(s)$. Define $\mathcal{D}($ point of $S)=$ Inv $u+\$_{1}-\operatorname{Inv} u-L\left(\$_{1}\right)$.

Consider $R$ being a function from the carrier of $S$ into the carrier of $W$ such that for every point $x$ of $S, R(x)=\mathcal{D}(x)$. For every point $x$ of $S, R(x)=\operatorname{Inv} u+x-\operatorname{Inv} u--(\operatorname{Inv} u) \cdot x \cdot(\operatorname{Inv} u)$. Reconsider $L_{0}=L$ as a point of the real norm space of bounded linear operators from $S$ into $W$. For every real number $r$ such that $r>0$ there exists a real number $d$ such that $d>0$ and for every point $z$ of $S$ such that $z \neq 0_{S}$ and $\|z\|<d$ holds $\|z\|^{-1} \cdot\left\|R_{/ z}\right\|<r$. Reconsider $R_{0}=R$ as a rest of $S, W$. For every point $v$ of $S$ such that $v \in N$ holds $I_{/ v}-I_{/ u}=L_{0}(v-u)+R_{0 / v-u}$.

(18) There exists a partial function $I$ from the real norm space of bounded linear operators from $X$ into $Y$ to the real norm space of bounded linear operators from $Y$ into $X$ such that

(i) $\operatorname{dom} I=\operatorname{InvertOpers}(X, Y)$, and

(ii) $\operatorname{rng} I=\operatorname{InvertOpers}(Y, X)$, and

(iii) $I$ is one-to-one and differentiable on $\operatorname{InvertOpers}(X, Y)$, and

(iv) there exists a partial function $J$ from the real norm space of bounded linear operators from $Y$ into $X$ to the real norm space of bounded linear operators from $X$ into $Y$ such that $J=I^{-1}$ and $J$ is one-to-one 
and $\operatorname{dom} J=\operatorname{InvertOpers}(Y, X)$ and $\operatorname{rng} J=\operatorname{InvertOpers}(X, Y)$ and $J$ is differentiable on InvertOpers $(Y, X)$, and

(v) for every point $u$ of the real norm space of bounded linear operators from $X$ into $Y$ such that $u \in \operatorname{InvertOpers}(X, Y)$ holds $I(u)=\operatorname{Inv} u$, and

(vi) for every points $u, d_{6}$ of the real norm space of bounded linear operators from $X$ into $Y$ such that $u \in \operatorname{InvertOpers}(X, Y)$ holds $\left(I^{\prime}(u)\right)\left(d_{6}\right)=-(\operatorname{Inv} u) \cdot d_{6} \cdot(\operatorname{Inv} u)$.

Proof: Consider $I$ being a partial function from the real norm space of bounded linear operators from $X$ into $Y$ to the real norm space of bounded linear operators from $Y$ into $X$ such that $\operatorname{dom} I=\operatorname{InvertOpers}(X, Y)$ and $\operatorname{rng} I=\operatorname{InvertOpers}(Y, X)$ and $I$ is one-to-one and continuous on InvertOpers $(X, Y)$ and there exists a partial function $J$ from the real norm space of bounded linear operators from $Y$ into $X$ to the real norm space of bounded linear operators from $X$ into $Y$ such that $J=I^{-1}$ and $J$ is oneto-one and $\operatorname{dom} J=\operatorname{InvertOpers}(Y, X)$ and $\operatorname{rng} J=\operatorname{InvertOpers}(X, Y)$ and $J$ is continuous on InvertOpers $(Y, X)$ and for every point $u$ of the real norm space of bounded linear operators from $X$ into $Y$ such that $u \in$ InvertOpers $(X, Y)$ holds $I(u)=\operatorname{Inv} u$.

Consider $J$ being a partial function from the real norm space of bounded linear operators from $Y$ into $X$ to the real norm space of bounded linear operators from $X$ into $Y$ such that $J=I^{-1}$ and $J$ is one-to-one and $\operatorname{dom} J=\operatorname{InvertOpers}(Y, X)$ and $\operatorname{rng} J=\operatorname{InvertOpers}(X, Y)$ and $J$ is continuous on InvertOpers $(Y, X)$. For every point $u$ of the real norm space of bounded linear operators from $X$ into $Y$ such that $u \in \operatorname{InvertOpers}(X, Y)$ holds $I$ is differentiable in $u$. For every point $v$ of the real norm space of bounded linear operators from $Y$ into $X$ such that $v \in \operatorname{InvertOpers}(Y, X)$ holds $J(v)=\operatorname{Inv} v$ by [5. (15)]. For every point $v$ of the real norm space of bounded linear operators from $Y$ into $X$ such that $v \in \operatorname{InvertOpers}(Y, X)$ holds $J$ is differentiable in $v$.

(19) Let us consider real normed spaces $E, G, F$, a subset $Z$ of $E \times F$, a partial function $f$ from $E \times F$ to $G$, a point $a$ of $E$, a point $b$ of $F$, a point $c$ of $G$, a point $z$ of $E \times F$, a subset $A$ of $E$, a subset $B$ of $F$, and a partial function $g$ from $E$ to $F$. Suppose $Z$ is open and $\operatorname{dom} f=Z$ and $A$ is open and $B$ is open and $A \times B \subseteq Z$ and $z=\langle a, b\rangle$ and $f(a, b)=c$ and $f$ is differentiable in $z$ and $\operatorname{dom} g=A$ and $\operatorname{rng} g \subseteq B$ and $a \in A$ and $g(a)=b$ and $g$ is continuous in $a$ and for every point $x$ of $E$ such that $x \in A$ holds $f(x, g(x))=c$ and partdiff $(f, z)$ w.r.t. 2 is invertible. Then

(i) $g$ is differentiable in $a$, and 
(ii) $g^{\prime}(a)=-(\operatorname{Inv} \operatorname{partdiff}(f, z)$ w.r.t. 2$) \cdot(\operatorname{partdiff}(f, z)$ w.r.t. 1$)$.

Proof: Consider $r_{2}$ being a real number such that $0<r_{2}$ and $\operatorname{Ball}\left(b, r_{2}\right) \subseteq$ $B$. Consider $r_{3}$ being a real number such that $0<r_{3}$ and for every point $x_{1}$ of $E$ such that $x_{1} \in \operatorname{dom} g$ and $\left\|x_{1}-a\right\|<r_{3}$ holds $\left\|g_{/ x_{1}}-g_{/ a}\right\|<r_{2}$. Consider $r_{4}$ being a real number such that $0<r_{4}$ and $\operatorname{Ball}\left(a, r_{4}\right) \subseteq A$. Set $r_{1}=\min \left(r_{3}, r_{4}\right)$. Set $g_{1}=g \uparrow \operatorname{Ball}\left(a, r_{1}\right)$. For every real number $r$ such that $0<r$ there exists a real number $s$ such that $0<s$ and for every point $x_{1}$ of $E$ such that $x_{1} \in \operatorname{dom} g_{1}$ and $\left\|x_{1}-a\right\|<s$ holds $\left\|g_{1 / x_{1}}-g_{1 / a}\right\|<r$. For every point $x$ of $E$ such that $x \in \operatorname{Ball}\left(a, r_{1}\right)$ holds $f\left(x, g_{1}(x)\right)=c$.

Reconsider $Q=$ (partdiff $(f, z)$ w.r.t. 2$)^{-1}$ as a Lipschitzian linear operator from $G$ into $F$. Reconsider $P=\operatorname{partdiff}(f, z)$ w.r.t. 1 as a Lipschitzian linear operator from $E$ into $G . g_{1}$ is differentiable in $a$ and $g_{1}{ }^{\prime}(a)=$ $-Q \cdot P$. Consider $N$ being a neighbourhood of $a$ such that $N \subseteq \operatorname{dom} g_{1}$ and there exists a rest $R$ of $E, F$ such that for every point $x$ of $E$ such that $x \in N$ holds $g_{1 / x}-g_{1 / a}=\left(g_{1}^{\prime}(a)\right)(x-a)+R_{/ x-a}$. Consider $R$ being a rest of $E, F$ such that for every point $x$ of $E$ such that $x \in N$ holds $g_{1 / x}-g_{1 / a}=\left(g_{1}^{\prime}(a)\right)(x-a)+R_{/ x-a}$. For every point $x$ of $E$ such that $x \in N$ holds $g_{/ x}-g_{/ a}=\left(g_{1}{ }^{\prime}(a)\right)(x-a)+R_{/ x-a}$.

(20) Let us consider a real normed space $E$, non trivial real Banach spaces $G, F$, a subset $Z$ of $E \times F$, a partial function $f$ from $E \times F$ to $G$, a point $c$ of $G$, a subset $A$ of $E$, a subset $B$ of $F$, and a partial function $g$ from $E$ to $F$. Suppose $Z$ is open and $\operatorname{dom} f=Z$ and $A$ is open and $B$ is open and $A \times B \subseteq Z$ and $f$ is differentiable on $Z$ and $f_{\uparrow Z}^{\prime}$ is continuous on $Z$ and $\operatorname{dom} g=A$ and $\operatorname{rng} g \subseteq B$ and $g$ is continuous on $A$ and for every point $x$ of $E$ such that $x \in A$ holds $f(x, g(x))=c$ and for every point $x$ of $E$ and for every point $z$ of $E \times F$ such that $x \in A$ and $z=\langle x, g(x)\rangle$ holds partdiff $(f, z)$ w.r.t. 2 is invertible. Then

(i) $g$ is differentiable on $A$, and

(ii) $g_{\uparrow A}^{\prime}$ is continuous on $A$, and

(iii) for every point $x$ of $E$ and for every point $z$ of $E \times F$ such that $x \in A$ and $z=\langle x, g(x)\rangle$ holds $g^{\prime}(x)=-(\operatorname{Inv} \operatorname{partdiff}(f, z)$ w.r.t. 2$)$.

(partdiff $(f, z)$ w.r.t. 1$)$.

Proof: For every point $x$ of $E$ and for every point $z$ of $E \times F$ such that $x \in A$ and $z=\langle x, g(x)\rangle$ holds $g$ is differentiable in $x$ and $g^{\prime}(x)=$ $-(\operatorname{Inv} \operatorname{partdiff}(f, z)$ w.r.t. 2$) \cdot(\operatorname{partdiff}(f, z)$ w.r.t. 1$)$. For every point $x$ of $E$ such that $x \in A$ holds $g$ is differentiable in $x$. Consider $x_{2}$ being a partial function from $E$ to $E \times F$ such that $\operatorname{dom} x_{2}=A$ and for every point $x$ of $E$ such that $x \in A$ holds $x_{2}(x)=\langle x, g(x)\rangle$ and $x_{2}$ is continuous on $A$. Consider $B$ being a bilinear operator from the real norm space of bounded 
linear operators from $E$ into $G \times$ the real norm space of bounded linear operators from $G$ into $F$ into the real norm space of bounded linear operators from $E$ into $F$ such that $B$ is continuous on the carrier of (the real norm space of bounded linear operators from $E$ into $G) \times($ the real norm space of bounded linear operators from $G$ into $F$ ) and for every point $u$ of the real norm space of bounded linear operators from $E$ into $G$ and for every point $v$ of the real norm space of bounded linear operators from $G$ into $F, B(u, v)=v \cdot u$.

Consider $I$ being a partial function from the real norm space of bounded linear operators from $F$ into $G$ to the real norm space of bounded linear operators from $G$ into $F$ such that $\operatorname{dom} I=\operatorname{InvertOpers}(F, G)$ and $\operatorname{rng} I=\operatorname{InvertOpers}(G, F)$ and $I$ is one-to-one and continuous on InvertOpers $(F, G)$ and there exists a partial function $J$ from the real norm space of bounded linear operators from $G$ into $F$ to the real norm space of bounded linear operators from $F$ into $G$ such that $J=I^{-1}$ and $J$ is one-toone and $\operatorname{dom} J=\operatorname{InvertOpers}(G, F)$ and $\operatorname{rng} J=\operatorname{InvertOpers}(F, G)$ and $J$ is continuous on $\operatorname{InvertOpers}(G, F)$ and for every point $u$ of the real norm space of bounded linear operators from $F$ into $G$ such that $u \in \operatorname{InvertOpers}(F, G)$ holds $I(u)=\operatorname{Inv} u$. For every point $x$ of $E$ such that $x \in A$ holds $\left(g_{\uparrow A}^{\prime}\right) / x=-B /\left\langle\left(\left(f \uparrow^{1} Z\right) \cdot x_{2}\right)(x),\left(I \cdot\left(f \uparrow^{2} Z\right) \cdot x_{2}\right)(x)\right\rangle$.

For every point $x$ of $E$ such that $x \in A$ holds $x \in \operatorname{dom}\left(\left(f \uparrow^{1} Z\right) \cdot x_{2}\right)$ and $\left(f \uparrow^{1} Z\right) \cdot x_{2}$ is continuous in $x$. For every point $x$ of $E$ such that $x \in A$ holds $x \in \operatorname{dom}\left(I \cdot\left(f \uparrow^{2} Z\right) \cdot x_{2}\right)$ and $I \cdot\left(f \uparrow^{2} Z\right) \cdot x_{2}$ is continuous in $x$. Consider $H$ being a partial function from $E$ to the real norm space of bounded linear operators from $E$ into $F$ such that $\operatorname{dom} H=A$ and for every point $x$ of $E$ such that $x \in A$ holds $H(x)=B\left(\left(\left(f \uparrow^{1} Z\right) \cdot x_{2}\right)(x),\left(I \cdot\left(f \uparrow^{2}\right.\right.\right.$ $\left.\left.Z) \cdot x_{2}\right)(x)\right)$ and $H$ is continuous on $A$. For every point $x_{0}$ of $E$ such that $x_{0} \in A$ holds $B\left(\left\langle\left(\left(f \uparrow^{1} Z\right) \cdot x_{2}\right)\left(x_{0}\right),\left(I \cdot\left(\left.f\right|^{2} Z\right) \cdot x_{2}\right)\left(x_{0}\right)\right\rangle\right)=$ $B /\left\langle\left(\left(f \uparrow^{1} Z\right) \cdot x_{2}\right)\left(x_{0}\right),\left(I \cdot\left(f \uparrow^{2} Z\right) \cdot x_{2}\right)\left(x_{0}\right)\right\rangle$. For every point $x_{0}$ of $E$ such that $x_{0} \in A$ holds $g_{\uparrow A}^{\prime}\left\lceil A\right.$ is continuous in $x_{0}$.

(21) Let us consider a real normed space $E$, non trivial real Banach spaces $G, F$, a subset $Z$ of $E \times F$, a partial function $f$ from $E \times F$ to $G$, a point $a$ of $E$, a point $b$ of $F$, a point $c$ of $G$, and a point $z$ of $E \times F$. Suppose $Z$ is open and $\operatorname{dom} f=Z$ and $f$ is differentiable on $Z$ and $f_{\uparrow Z}^{\prime}$ is continuous on $Z$ and $\langle a, b\rangle \in Z$ and $f(a, b)=c$ and $z=\langle a, b\rangle$ and partdiff $(f, z)$ w.r.t. 2 is invertible. Then there exist real numbers $r_{1}, r_{2}$ such that

(i) $0<r_{1}$, and

(ii) $0<r_{2}$, and

(iii) $\operatorname{Ball}\left(a, r_{1}\right) \times \overline{\operatorname{Ball}}\left(b, r_{2}\right) \subseteq Z$, and 
(iv) for every point $x$ of $E$ such that $x \in \operatorname{Ball}\left(a, r_{1}\right)$ there exists a point $y$ of $F$ such that $y \in \operatorname{Ball}\left(b, r_{2}\right)$ and $f(x, y)=c$, and

(v) for every point $x$ of $E$ such that $x \in \operatorname{Ball}\left(a, r_{1}\right)$ for every points $y_{1}, y_{2}$ of $F$ such that $y_{1}, y_{2} \in \operatorname{Ball}\left(b, r_{2}\right)$ and $f\left(x, y_{1}\right)=c$ and $f\left(x, y_{2}\right)=c$ holds $y_{1}=y_{2}$, and

(vi) there exists a partial function $g$ from $E$ to $F$ such that $\operatorname{dom} g=$ $\operatorname{Ball}\left(a, r_{1}\right)$ and $\operatorname{rng} g \subseteq \operatorname{Ball}\left(b, r_{2}\right)$ and $g$ is continuous on $\operatorname{Ball}\left(a, r_{1}\right)$ and $g(a)=b$ and for every point $x$ of $E$ such that $x \in \operatorname{Ball}\left(a, r_{1}\right)$ holds $f(x, g(x))=c$ and $g$ is differentiable on $\operatorname{Ball}\left(a, r_{1}\right)$ and $g_{\mid \operatorname{Ball}\left(a, r_{1}\right)}^{\prime}$ is continuous on $\operatorname{Ball}\left(a, r_{1}\right)$ and for every point $x$ of $E$ and for every point $z$ of $E \times F$ such that $x \in \operatorname{Ball}\left(a, r_{1}\right)$ and $z=\langle x, g(x)\rangle$ holds $g^{\prime}(x)=-(\operatorname{Inv}$ partdiff $(f, z)$ w.r.t. 2$) \cdot($ partdiff $(f, z)$ w.r.t. 1$)$ and for every point $x$ of $E$ and for every point $z$ of $E \times F$ such that $x \in$ $\operatorname{Ball}\left(a, r_{1}\right)$ and $z=\langle x, g(x)\rangle$ holds partdiff $(f, z)$ w.r.t. 2 is invertible, and

(vii) for every partial functions $g_{1}, g_{2}$ from $E$ to $F$ such that $\operatorname{dom} g_{1}=$ $\operatorname{Ball}\left(a, r_{1}\right)$ and $\operatorname{rng} g_{1} \subseteq \operatorname{Ball}\left(b, r_{2}\right)$ and for every point $x$ of $E$ such that $x \in \operatorname{Ball}\left(a, r_{1}\right)$ holds $f\left(x, g_{1}(x)\right)=c$ and $\operatorname{dom} g_{2}=\operatorname{Ball}\left(a, r_{1}\right)$ and $\operatorname{rng} g_{2} \subseteq \operatorname{Ball}\left(b, r_{2}\right)$ and for every point $x$ of $E$ such that $x \in$ $\operatorname{Ball}\left(a, r_{1}\right)$ holds $f\left(x, g_{2}(x)\right)=c$ holds $g_{1}=g_{2}$.

Proof: Set $P=f_{0} \uparrow^{2} Z_{0}$. Consider $p_{1}$ being a real number such that $0<p_{1}$ and $\operatorname{Ball}\left(P_{/ z}, p_{1}\right) \subseteq \operatorname{InvertOpers}(F, G)$. Consider $s_{1}$ being a real number such that $0<s_{1}$ and for every point $z_{1}$ of $E \times F$ such that $z_{1} \in Z_{0}$ and $\left\|z_{1}-z\right\|<s_{1}$ holds $\left\|P_{/ z_{1}}-P_{/ z}\right\|<p_{1}$. Consider $s_{2}$ being a real number such that $0<s_{2}$ and $\operatorname{Ball}\left(z, s_{2}\right) \subseteq Z_{0}$. Set $s=\min \left(s_{1}, s_{2}\right)$. Set $Z=\operatorname{Ball}(z, s)$. Set $f=f_{0} \uparrow Z$. Set $D=f_{\uparrow Z}^{\prime}$. For every point $z$ of $E \times$ $F$ such that $z \in Z$ holds $f_{0}^{\prime}(z)=f^{\prime}(z)$. For every point $x_{0}$ of $E \times F$ and for every real number $r$ such that $x_{0} \in Z$ and $0<r$ there exists a real number $s$ such that $0<s$ and for every point $x_{1}$ of $E \times F$ such that $x_{1} \in Z$ and $\left\|x_{1}-x_{0}\right\|<s$ holds $\left\|D_{/ x_{1}}-D_{/ x_{0}}\right\|<r$. For every point $z$ of $E \times F$ such that $z \in Z$ holds partdiff $\left(f_{0}, z\right)$ w.r.t. $1=\operatorname{partdiff}(f, z)$ w.r.t. 1 and $\operatorname{partdiff}\left(f_{0}, z\right)$ w.r.t. $2=\operatorname{partdiff}(f, z)$ w.r.t. 2 .

Consider $r_{1}, r_{2}$ being real numbers such that $0<r_{1}$ and $0<r_{2}$ and $\operatorname{Ball}\left(a, r_{1}\right) \times \overline{\operatorname{Ball}}\left(b, r_{2}\right) \subseteq Z$ and for every point $x$ of $E$ such that $x \in \operatorname{Ball}\left(a, r_{1}\right)$ there exists a point $y$ of $F$ such that $y \in \operatorname{Ball}\left(b, r_{2}\right)$ and $f(x, y)=c$ and for every point $x$ of $E$ such that $x \in \operatorname{Ball}\left(a, r_{1}\right)$ for every points $y_{1}, y_{2}$ of $F$ such that $y_{1}, y_{2} \in \operatorname{Ball}\left(b, r_{2}\right)$ and $f\left(x, y_{1}\right)=c$ and $f\left(x, y_{2}\right)=c$ holds $y_{1}=y_{2}$ and there exists a partial function $g$ from $E$ to $F$ such that $g$ is continuous on $\operatorname{Ball}\left(a, r_{1}\right)$ and $\operatorname{dom} g=\operatorname{Ball}\left(a, r_{1}\right)$ and 
$\operatorname{rng} g \subseteq \operatorname{Ball}\left(b, r_{2}\right)$ and $g(a)=b$ and for every point $x$ of $E$ such that $x \in \operatorname{Ball}\left(a, r_{1}\right)$ holds $f(x, g(x))=c$ and for every partial functions $g_{1}, g_{2}$ from $E$ to $F$ such that $\operatorname{dom} g_{1}=\operatorname{Ball}\left(a, r_{1}\right)$ and $\operatorname{rng} g_{1} \subseteq \operatorname{Ball}\left(b, r_{2}\right)$ and for every point $x$ of $E$ such that $x \in \operatorname{Ball}\left(a, r_{1}\right)$ holds $f\left(x, g_{1}(x)\right)=c$ and $\operatorname{dom} g_{2}=\operatorname{Ball}\left(a, r_{1}\right)$ and $\operatorname{rng} g_{2} \subseteq \operatorname{Ball}\left(b, r_{2}\right)$ and for every point $x$ of $E$ such that $x \in \operatorname{Ball}\left(a, r_{1}\right)$ holds $f\left(x, g_{2}(x)\right)=c$ holds $g_{1}=g_{2}$.

For every point $x$ of $E$ and for every point $y$ of $F$ such that $x \in$ $\operatorname{Ball}\left(a, r_{1}\right)$ and $y \in \operatorname{Ball}\left(b, r_{2}\right)$ holds $f_{0}(x, y)=f(x, y)$. For every point $x$ of $E$ such that $x \in \operatorname{Ball}\left(a, r_{1}\right)$ there exists a point $y$ of $F$ such that $y \in$ $\operatorname{Ball}\left(b, r_{2}\right)$ and $f_{0}(x, y)=c$. For every point $x$ of $E$ such that $x \in \operatorname{Ball}\left(a, r_{1}\right)$ for every points $y_{1}, y_{2}$ of $F$ such that $y_{1}, y_{2} \in \operatorname{Ball}\left(b, r_{2}\right)$ and $f_{0}\left(x, y_{1}\right)=c$ and $f_{0}\left(x, y_{2}\right)=c$ holds $y_{1}=y_{2}$. Consider $g$ being a partial function from $E$ to $F$ such that $g$ is continuous on $\operatorname{Ball}\left(a, r_{1}\right)$ and $\operatorname{dom} g=\operatorname{Ball}\left(a, r_{1}\right)$ and $\operatorname{rng} g \subseteq \operatorname{Ball}\left(b, r_{2}\right)$ and $g(a)=b$ and for every point $x$ of $E$ such that $x \in \operatorname{Ball}\left(a, r_{1}\right)$ holds $f(x, g(x))=c$. For every point $x$ of $E$ and for every point $w$ of $E \times F$ such that $x \in \operatorname{Ball}\left(a, r_{1}\right)$ and $w=\langle x, g(x)\rangle$ holds partdiff $\left(f_{0}, w\right)$ w.r.t. 2 is invertible. For every point $x$ of $E$ and for every point $w$ of $E \times F$ such that $x \in \operatorname{Ball}\left(a, r_{1}\right)$ and $w=\langle x, g(x)\rangle$ holds partdiff $(f, w)$ w.r.t. 2 is invertible.

For every point $x$ of $E$ such that $x \in \operatorname{Ball}\left(a, r_{1}\right)$ holds $f_{0}(x, g(x))=c$. $g$ is differentiable on $\operatorname{Ball}\left(a, r_{1}\right)$ and $g_{\uparrow \operatorname{Ball}\left(a, r_{1}\right)}^{\prime}$ is continuous on $\operatorname{Ball}\left(a, r_{1}\right)$ and for every point $x$ of $E$ and for every point $z$ of $E \times F$ such that $x \in$ $\operatorname{Ball}\left(a, r_{1}\right)$ and $z=\langle x, g(x)\rangle$ holds $g^{\prime}(x)=-\left(\operatorname{Inv} \operatorname{partdiff}\left(f_{0}, z\right)\right.$ w.r.t. 2$)$. (partdiff $\left(f_{0}, z\right)$ w.r.t. 1$)$. For every partial functions $g_{1}, g_{2}$ from $E$ to $F$ such that $\operatorname{dom} g_{1}=\operatorname{Ball}\left(a, r_{1}\right)$ and $\operatorname{rng} g_{1} \subseteq \operatorname{Ball}\left(b, r_{2}\right)$ and for every point $x$ of $E$ such that $x \in \operatorname{Ball}\left(a, r_{1}\right)$ holds $f_{0}\left(x, g_{1}(x)\right)=c$ and $\operatorname{dom} g_{2}=\operatorname{Ball}\left(a, r_{1}\right)$ and $\operatorname{rng} g_{2} \subseteq \operatorname{Ball}\left(b, r_{2}\right)$ and for every point $x$ of $E$ such that $x \in \operatorname{Ball}\left(a, r_{1}\right)$ holds $f_{0}\left(x, g_{2}(x)\right)=c$ holds $g_{1}=g_{2}$.

\section{REFERENCES}

[1] Grzegorz Bancerek, Czesław Byliński, Adam Grabowski, Artur Korniłowicz, Roman Matuszewski, Adam Naumowicz, and Karol Pąk. The role of the Mizar Mathematical Library for interactive proof development in Mizar. Journal of Automated Reasoning, 61(1):9-32, 2018. do1:10.1007/s10817-017-9440-6

[2] Bruce K. Driver. Analysis Tools with Applications. Springer, Berlin, 2003.

[3] Adam Grabowski, Artur Korniłowicz, and Adam Naumowicz. Four decades of Mizar. Journal of Automated Reasoning, 55(3):191-198, 2015. doi 10.1007/s10817-015-9345-1

[4] Hiroshi Imura, Morishige Kimura, and Yasunari Shidama. The differentiable functions on normed linear spaces Formalized Mathematics, 12(3):321-327, 2004.

[5] Kazuhisa Nakasho. Invertible operators on Banach spaces. Formalized Mathematics, 27 (2):107-115, 2019. doi 10.2478/forma-2019-0012.

[6] Kazuhisa Nakasho, Yuichi Futa, and Yasunari Shidama. Implicit function theorem. Part I. Formalized Mathematics, 25(4):269-281, 2017. doi:10.1515/forma-2017-0026. 
[7] Takaya Nishiyama, Keiji Ohkubo, and Yasunari Shidama. The continuous functions on normed linear spaces Formalized Mathematics, 12(3):269-275, 2004.

[8] Hiroyuki Okazaki, Noboru Endou, and Yasunari Shidama. Cartesian products of family of real linear spaces. Formalized Mathematics, 19(1):51-59, 2011. doi:10.2478/v10037011-0009-2.

[9] Hideki Sakurai, Hiroyuki Okazaki, and Yasunari Shidama. Banach's continuous inverse theorem and closed graph theorem. Formalized Mathematics, 20(4):271-274, 2012. doi: $10.2478 /$ v10037-012-0032-y

[10] Laurent Schwartz. Théorie des ensembles et topologie, tome 1. Analyse. Hermann, 1997.

[11] Laurent Schwartz. Calcul différentiel, tome 2. Analyse. Hermann, 1997.

Accepted May 27, 2019 